\title{
Influência da atividade física na imagem corporal: Uma revisão bibliográfica
}

\author{
The influence of physical activity on body image: A literature review
}

\author{
C.O. Gonçalves, A.N. Campana, M.C. Tavares
}

ARTIGO DE REVISÃO | REVIEW ARTICLE

\begin{abstract}
A imagem corporal pode ser entendida como a representação mental do nosso próprio corpo. Sendo a atividade física uma fonte de vivências corporais, faz-se necessário ter uma visão ampliada de seus efeitos sobre a imagem corporal. O objetivo deste trabalho foi analisar a evolução da pesquisa em imagem corporal e atividade física, assim como desenvolver uma reflexão sobre as relações existentes entre a atividade física e a imagem corporal. Foram utilizadas as bases de dados - Web of Science, Scopus e SportDiscus. Um total de 10 estudos foi criteriosamente selecionado. A análise dos estudos aponta para uma evolução significativa dos desenhos dessas pesquisas, um importante desenvolvimento da metodologia utilizada e dos recursos de avaliação. Além disso, conclui-se que a atividade física exerce influências na imagem corporal de diversas formas. Um programa de atividade física deve respeitar a individualidade do sujeito para determinar intensidade, frequência e duração adotados, sabendo que as indicações mínimas para obtenção de repostas relativas ao desenvolvimento da imagem corporal são de duas sessões de treino por semana, durante 12 semanas.
\end{abstract}

Palavras-chave: imagem corporal, atividade física, revisão de literatura

ABSTRACT

Body image can be understood as the mental representation of our own body. Since physical activity is a source of bodily experiences, it is necessary to have a wider view of their effects on body image. The aim of this study was to analyze the evolution of research on body image and physical activity and to reflect about the relationship between physical activity and body image. We used three databases - Web of Science, Scopus and SportDiscus. A total of 10 studies were carefully selected. The review points to a significant evolution of the designs of these surveys, an important development of methodology and evaluation resources. Moreover, it is concluded that physical activity exerts influences on body image in various ways. A program of physical activity must respect the person individuality to determine the adopted intensity, frequency and length, knowing that the minimal recommendations to achieve a feedback related with body image development regard to two sessions per week during 12 weeks.

Keywords: body image, physical activity, literature review

Submetido: 02.04.2010 | Aceite: 09.04.2011 | Online:

Carolina de Oliveira Gonçalves. Mestre em Educação Física, Adaptação e Saúde - FEF/UNICAMP, Campinas/SP, Brasil.

Angela Nogueira Campana. Doutora em Educação Física. Participante do Grupo de Estudos e Pesquisa em Imagem Corporal e pesquisadora no Laboratório de Imagem Corporal - FEF/UNICAMP, Campinas/SP, Brasil.

Maria da Consolação Tavares. Professora Titular do Departamento de Estudos de Atividade Física Adaptada FEF/UNICAMP; Líder do Grupo de Estudos e Pesquisa em Imagem Corporal e docente responsável pelo Laboratório de Imagem Corporal - FEF/UNICAMP, Campinas/SP, Brasil.

Endereço para correspondência: Carolina de Oliveira Gonçalves, Laboratório de Imagem Corporal. Universidade Estadual de Campinas, Faculdade de Educação Física, Laboratório de Imagem Corporal, Av. Érico Veríssimo, 701, CEP: 13083-851, Campinas, SP, Brasil.

E-mail: carol.fef06d@gmail.com 
A imagem corporal pode ser entendida com a representação mental do corpo. Ela é dinâmica e singular. Este constructo multifacetado tem componentes percetivos e atitudinais cognitivos, emocionais e comportamentais que se estabelecem num continuum, partindo de valores positivos e usualmente associados a sentimentos e comportamentos saudáveis prática de exercícios, cuidados com o corpo, relações sociais estáveis, autoestima - para valores negativos, associados a outros quadros clínicos - como depressão e obesidade, por exemplo. Estes valores negativos associam-se a distúrbios da representação do corpo, estando alguns descritos nos manuais de diagnóstico de doenças mentais - i.e., transtorno alimentar e dismorfia corporal - ou com evidências fortes apontando para uma relação causal - i.e., dismorfia muscular (Cash, 2004; Hausenblas \& Fallon, 2006).

Os problemas com a imagem do corpo podem progredir de uma moderada insatisfação para uma preocupação extrema com a aparência física, levando a uma imagem corporal negativa, que é uma condição mais estressante e inibitória que a insatisfação corporal inicial (Rosen, Orosan, \& Reiter, 1995). Cash (2004) apresenta a imagem corporal negativa como um forte sentimento de insatisfação com aspetos da aparência física que as pessoas têm consigo mesmas. Historicamente as pesquisas em imagem corporal focaram-se em avaliar, descobrir relações, estabelecer causas e tratamentos para a imagem corporal negativa (Thompson \& Gardner, 2002). Neste processo variadas formas de intervenção foram investigadas com o intuito de avaliar seus efeitos, ou descobrir variáveis correlacionadas que fornecessem evidências sobre como promover compensações longitudinais a fim de propiciar o desenvolvimento da imagem corporal (Cash \& Pruzinsky, 2002; Thompson, 1990). Com este intuito, o número de estudos sobre o exercício no tratamento da saúde mental e na promoção do bem-estar vem crescendo e importantes informações sobre a influência da atividade física sistematizada na imagem corporal estão sendo descobertas e.g., a relação entre o tempo de prática e melhorias na auto-estim. Esclarecemos que, segundo o estudo de Fox e Wilson (2008) o tempo mínimo de duração de um programa de atividade física/exercícios não deve ser inferior a um período de 12 semanas, pois um tempo inferior a este pode não ser suficiente para mudar um constructo, especialmente quando este é suscetível a outros eventos que ocorrem simultaneamente na vida.

Entretanto, nem sempre a atividade física é usada na busca para estabelecer uma imagem corporal bem estruturada. $\mathrm{O}$ abuso do exercício - atividade física sistematizada, que possui frequência, duração e intensidade delineadas (Caspersen, Powell, \& Christensen, 1985), tem sido motivo de frequente preocupação nos casos de dismorfia corporal, pelo alto índice de dependência que pode gerar. Outra preocupação é o uso do exercício como método compensatório nos quadros de bulimia nervosa. Esses dois exemplos ilustram como a atividade física pode auxiliar na perpetuação de distúrbios, que envolvem uma representação prejudicada do corpo (Assunção, Cordas, \& Araújo, 2002).

Para Tavares (2003), a atividade física (incluindo o exercício) deve proporcionar ao indivíduo vivências que possibilitem um desenvolvimento de sua imagem corporal, o que implica, em última instância, tornar-se consciente de seus próprios sentimentos e reações fisiológicas em relação ao corpo e à atividade, respeitando seus limites e suas possibilidades. Este é um processo que pode ser facilitado pelo profissional da educação física ao trabalhar experiências corporais com seus alunos, considerando as necessidades educativas especiais dos mesmos (Tavares, 2007).

Dada a possibilidade de ser uma fonte de compensações longitudinais e também de agravamento de transtornos, é de extrema importância não só ao profissional de educação física, mas a todos os profissionais da saúde, o conhecimento das consequências que algumas 
modalidades de atividades físicas podem exercer na imagem corporal. Assim sendo, os objetivos deste trabalho foram: (a) analisar as características e a evolução da pesquisa em imagem corporal e atividade física, e (b) desenvolver uma reflexão sobre as relações entre atividade física e imagem corporal, identificando os efeitos que diferentes tipos de atividade, intensidade, duração e frequência podem exercer na imagem corporal. Para este fim, reunimos trabalhos experimentais realizados nos últimos 30 anos que avaliaram os efeitos na imagem corporal de pessoas submetidas à intervenção de atividades físicas sistematizadas.

\section{MÉTODO}

Esta pesquisa é uma revisão de bibliografia através da qual foi feita a análise, avaliação crítica e a integração da literatura publicada sobre imagem corporal e atividade física.

Os dados foram reunidos a partir de três bases internacionais: Web of Science, Scopus e SportDiscuss. Nas três bases, foram combinadas as palavras-chave body image, longitudinal, exercise, physical activity, physical exercise, fitness, exercise training com o operador booleano AND.

Como critério de inclusão adotou-se selecionar (a) artigos publicados nos últimos trinta anos, (b) estudos experimentais ou semi-experimentais, (c) estudos de natureza qualitativa ou quantitativa, (d) estudos que contivessem descrição da intervenção de atividade física realizada, (e) intervenção com duração mínima de 12 semanas (seguindo as recomendações de Fox \& Wilson, 2008), e, por fim, (f) os estudos poderiam incluir a população em geral (ambos os sexos, idade e situações especiais, e.g.: gravidez). Por sua vez, foram excluídos deste estudo os artigos que (a) não realizaram intervenção de atividades físicas ou que as realizaram em um período de tempo inferior a 12 semanas; (b) estudos que não descreveram as atividades realizadas na intervenção, e (c) estudos que não usaram questionários validados em sua coleta de dados, no caso de estudos quantitativos.

\section{RESULTADOS}

Foram encontrados na pesquisa bibliográfica um total de 107 artigos. Deste total, 29 artigos foram excluídos por aparecerem repetidamente nas bases de dados, configurando 78 artigos exclusivos. Após aplicados os critérios de inclusão e exclusão, chegámos ao resultado final deste levantamento bibliográfico, para o qual foram selecionados um total de 10 artigos.

Para fins de análise e melhor compreensão do leitor, os artigos serão apresentados nas seguintes subcategorias, dentro da categoria imagem corporal e atividade física: crianças asmáticas, mulheres e grupos de ambos os sexos.

\section{Imagem corporal e atividade física: Crianças asmáticas}

Apenas um dos estudos teve como foco a população de crianças. Varray (1988) investigou os efeitos de exercícios recreativos na água, com crianças asmáticas entre sete e 10 anos. As aulas tiveram duração de 24 semanas e freqüência de uma a duas horas semanais. Os testes e questionários utilizados foram: (a) Body Image Questionnaire (Bruchon-Schweitzer, 1987), (b) Inventário de Autoestima (Coopersmith, 1971), (c) Teste Projetivo de Rorschach, e (d) entrevista semiestruturada com os pais. Os resultados mostraram que após a prática da natação houve melhor reconhecimento do corpo em sua totalidade, diminuição do quadro de ansiedade assim como diminuição dos escores barreira/penetração (para mais informações, ver Fisher \& Cleveland, 1958). Entretanto, o autor chama atenção para um aspeto metodológico desta pesquisa: a seleção dos testes utilizados. Apesar das escalas psicométricas selecionadas terem propriedades psicométricas adequadas para a população - validade e confiabilidade - o único teste que identificou alterações na população estudada foi o teste projetivo de Rorschach. A este fato, Varray (1988) atribuiu duas hipóteses: os outros testes não foram sensíveis o suficiente ou o número amostral reduzido influenciou negativamente os resultados. A entrevista 
realizada com os pais, ao contrário do que se esperava, não mostrou que era capaz de influenciar a imagem corporal das crianças.

\section{Imagem corporal e atividade física: Mulheres}

Dos 10 artigos analisados, seis trabalharam exclusivamente com a população feminina. Vandereycken, Depreitere, e Probst (1987) realizaram um estudo com o propósito de influenciar a maneira como jovens mulheres em tratamento para anorexia nervosa experienciavam seus corpos. A intervenção, que teve uma duração total de quatro anos, consistiu em uma terapia corporal orientada, com variadas técnicas corporais - relaxamentos, exercícios bioenergéticos, expressão livre na dança, grupos de comunicação não verbal, entre outras atividades realizadas em intensidades leves. A avaliação do tratamento foi realizada através de entrevistas, questionários - Eating Disorder Inventory (Garner, Olmstead, \& Polivy, 1983) e Body Attitudes Test (Vandereycken \& Meermann, 1984) - vídeos, encontros e discussões em grupo. Os resultados indicaram que além do aumento do peso, aos poucos, as pacientes demonstram prazer em realizar as atividades, conseguiram relaxar e expressar seus sentimentos com mais facilidade, se sentiram mais motivadas, aumentaram a capacidade de perceção corporal e desenvolveram uma imagem corporal positiva.

Tucker e Maxwell (1992) publicaram uma pesquisa sobre os efeitos do treinamento de peso no bem-estar emocional e na imagem corporal de mulheres. Participaram do estudo 152 mulheres com média de idade igual a 20.2 anos, todas universitárias, divididas em grupo experimental e grupo de controle. Os sujeitos de ambos os grupos responderam, pré e pósintervenção, aos questionários (a) General Well Being Schedule (Dupuy, 1970) - que avalia o bem-estar subjetivo e a angústia psicológica - e (b) a uma versão modificada da Body Cathexis Scale (modificada por Tucker, 1981) - que avalia a satisfação com partes do corpo. Além dos questionários, os sujeitos do grupo experimental fizeram também testes de força muscular (1 RM) e medidas de três dobras cutâneas.

O grupo experimental foi submetido a 12 semanas de treinamento com pesos. O treino foi realizado duas vezes por semana, com duração de aproximadamente 45 minutos. A intensidade foi moderada, com três séries de 10 repetições para cada exercício, a saber: supino reto, rosca direta com halteres, leg press sentado na máquina. Ao final da intervenção as mulheres do grupo experimental apresentaram perda de peso, significativo aumento de força muscular e queda nas medidas de dobras cutâneas. Os resultados dos testes General WellBeing e Body Cathexis Scale permitiram inferir que a intervenção teve um efeito positivo sobre a imagem corporal, pois quando comparado ao grupo de controle, o grupo experimental apresentou escores mais positivos.

No ano seguinte, Tucker e Mortell (1993) compararam os efeitos da caminhada e do treinamento de peso na imagem corporal de mulheres de meia-idade. Participaram do estudo 60 mulheres com média de idade igual a 42.5 anos. Os sujeitos foram separados aleatoriamente em dois grupos: treinamento resistido ou caminhada. Ambos os grupos fizeram as atividades três dias por semana, durante 12 semanas. Para avaliar os efeitos do exercício, os pesquisadores utilizaram a Body Cathexis Scale (Tucker, 1981) e realizaram testes de 1RM e testes de caminhada de 1 milha. Os resultados antes e pós-intervenção indicaram que o treinamento regular resistido foi estatisticamente significante no aumento da força muscular dinâmica e absoluta. Os exercícios regulares de caminhada provocaram aumento significante na resistência cardiorrespiratória ao longo de três meses de experimento. Além da confirmação dos resultados esperados, ocorreram mudanças na imagem corporal aumento da satisfação com o corpo, em ambos os grupos. Comparando os grupos entre si, os autores verificaram que o grupo de treinamento resistido teve maiores ganhos de força muscular, assim como melhorias significantes na imagem corporal, quando comparados ao 
grupo de caminhada. Para os autores, o treino resistido causou os melhores resultados na imagem corporal por promover as mudanças mais visíveis na aparência do corpo quando comparado ao treino de caminhada. Enquanto os efeitos da caminhada são menos visíveis aparentemente, o treino resistido torna os músculos mais tonificados, mais definidos e isso pode ser um mecanismo de feedback positivo que reforça a autoestima e aumenta a satisfação com o corpo.

Anos mais tarde, Stoll (2000) realizou um estudo quase-experimental sobre aptidão física e as estimativas de recursos, focado em avaliar os efeitos das variáveis psicossociais de um programa de atividade física moderada. A intervenção teve duração de 12 semanas, com frequência de uma vez por semana, duração entre 60 e 75 minutos, e contou com exercícios variados, em intensidade moderada, assim de melhorar a flexibilidade e a força. Vinte e uma mulheres (média de idade igual a 34.14 anos), sedentárias há pelo menos dois anos, foram distribuídas aleatoriamente por um grupo experimental $(n=10)$ que participou da intervenção e um grupo de controle $(n=11)$, que não fez atividades durante este período. $\mathrm{O}$ conceito do corpo, o autoconceito sobre desempenho e o autoconceito na comunicação e nas competências pessoais foram avaliados, respectivamente, através da escala Köperkonzept (KSK) de Alfermann e Stoll (1996), e das subescalas Selbstkonzepts zur Allgemeinen Leistungsfähigkeit (FSAL) e Selbstkonzepts zur Kommunikations - und Umgangsfähigkeit (FSKU), ambas pertencentes à escala Frankfurter Selbstkonzeptskalen (FSK), desenvolvida por Deusinger (1986). Os questionários foram preenchidos em três momentos: (T1) antes do início da intervenção, (T2) logo após o término da intervenção, e (T3) 12 semanas após o término das intervenções.

A análise dos resultados mostrou que o grupo experimental, quando comparado ao grupo de controle, apresentou melhorias significantes referentes às variáveis autoconceito, perceção da aptidão e atitude negativa em relação ao corpo. Antes do programa de exercícios, os participantes do grupo de controle obtiveram resultados mais baixos para as variáveis imagem corporal negativa e preocupações com o físico, quando comparados ao grupo experimental. Foram observadas mudanças significativas no autoconceito, na perceção da aptidão, na ansiedade e na autoestima dos participantes do grupo experimental, após a atividade física. A ansiedade diminuiu progressivamente de $\mathrm{T} 1$ a $\mathrm{T} 3$, enquanto o recurso autoestima aumentou continuamente em todas as três medidas. Já as outras duas variáveis perceção da aptidão e autoconceito - obtiveram resultados positivos de $\mathrm{T} 1$ a $\mathrm{T} 2$, mas tiveram quedas após a suspensão dos exercícios (T3). Assim, pode-se afirmar a contribuição da atividade física moderada para a melhoria da conceção do corpo. Contudo, algumas alterações, como perceção da aptidão, podem não ser sustentadas com o cessar das atividades físicas.

Em um estudo com mulheres com câncer de mama, Pinto, Clark, Maruyama e Feder (2003) analisaram as mudanças psicológicas e físicas dessas mulheres associadas à participação ao exercício físico. Participaram do estudo 24 mulheres sedentárias, diagnosticadas com câncer de mama nos três anos anteriores ao estudo, e que tiveram suas sessões de quimioterapia e radioterapia (póscirurgia) terminadas. Essas mulheres foram divididas em grupo experimental - submetido a 12 semanas exercícios aeróbios moderados (60-70\% da frequência cardíaca máxima), três vezes por semana - e grupo de controle - não praticou exercícios. Para a avaliação do programa foi utilizado o teste de pico ergômetro e três questionários: (a) o Profile of Mood States (POMS; McNair, Lorr, \& Droppelman, 1971), (b) a Positive and Negative Affect Scale (PANAS; Watson, Clark, \& Tellegen, 1988), e (c) a Body Esteem Scale (BES; Franzoi \& Shields, 1984).

Os resultados indicaram que o programa de intervenção melhorou a imagem corporal das participantes do grupo experimental nas subescalas de Condições Físicas e Preocupações com o Peso da Body Esteem Scale. O grupo de contro- 
le relatou uma diminuição nos escores dessas subescalas, reforçando o papel que o exercício pode ter na prevenção de declínios na satisfação com a imagem corporal de pacientes com câncer. O grupo experimental mostrou uma diminuição dos sentimentos de angústia, verificada na POMS e PANAS, mas esses resultados não foram considerados confiáveis, uma vez que o $n$ da amostra foi baixo (e diminuído durante a pesquisa - três mulheres do grupo experimental desistiram da pesquisa).

Calogero e Pedrotty (2004) investigaram os efeitos de um programa intervenção física projetado para reduzir o abuso de exercícios em mulheres com distúrbios alimentares. Participaram do estudo 254 mulheres, diagnosticadas com anorexia nervosa subtipo restritivo $(n=82)$, anorexia nervosa subtipo purgativo $(n=33)$, bulimia nervosa $(n=89)$, ou transtorno alimentar não especificado $(n=$ 50), admitidas para tratamento durante um período de seis meses. A amostra foi dividida em grupos experimental - que praticaram exercícios por pelo menos duas vezes por semana - e de controle, que não fez exercício físico. Para avaliação dos efeitos da intervenção sobre a saúde mental e física das pacientes, os pesquisadores utilizaram (a) uma versão adaptada do Exercise History and Eating Disorder Severity, a partir da $12^{\mathrm{a}}$ edição do Eating Disorders Examination (Fairburn \& Wilson, 1993), (b) o Obligatory Exercise Questionnaire (Thompson \& Pasman, 1991), (c) a subescala Appearance Control da escala Objectified Body Consciousness (McKinley \& Hyde, 1996), e (d) o Eating Disorder Patient's Expectations and Experiences of Treatment (Clinton, 2001). Adicionalmente, registraram o ganho de peso semanal e total das pacientes. A intervenção consistiu num programa de exercícios de coordenação, alongamento, exercícios posturais, treino de flexibilidade, equilíbrio, jogos recreativos ou outras atividades que elas gostassem. As atividades foram realizadas quatro vezes na semana, com uma duração de 60 minutos por sessão.

Após a análise dos resultados, concluiu-se que as mulheres diagnosticadas com anorexia nervosa subtipo restritivo e anorexia nervosa subtipo purgativo que participaram da intervenção aumentaram seu peso em cerca de $40 \%$ quando comparadas às pacientes com o mesmo diagnóstico do grupo de controle. Como esperado, o grupo diagnosticado com bulimia nervosa não teve alterações significativas no peso em relação ao grupo de controle. Já o grupo de transtorno alimentar não especificado não sofreu alterações em relação ao peso, contrariando o que era esperado para o grupo. Comparando os resultados antes e após a intervenção Calogero e Pedrotty (2004) observaram que as participantes do grupo experimental demonstraram uma redução significativa na ansiedade, e diminuíram seu comprometimento emocional, envolvimento e rigidez com o exercício. Além disso, elas mudaram a opinião sobre exercício e saúde ao deixarem de lado algumas das falsas crenças que tinham sobre o assunto. Para os autores, essas descobertas sugerem que o uso de um programa que tenha como meta a redução do abuso de exercícios em mulheres com transtornos alimentares é possível durante o tratamento residencial e resulta em mudanças positivas sem interferir no ganho de peso.

\section{Imagem corporal e atividade física: Ambos os sexos}

Nenhum dos artigos selecionados de acordo com os critérios de inclusão e exclusão, desta revisão, estudou os efeitos da atividade física na imagem corporal exclusivamente na população masculina. Dados a respeito da população masculina apareceram apenas em três pesquisas com populações mistas.

Alfermann e Stoll (2000) investigaram os efeitos do exercício físico no autoconceito e no bem-estar de adultos de meia-idade de ambos os sexos, sedentários há pelo menos 12 meses. Esta pesquisa consta de dois estudos, o primeiro com um grupo experimental e um grupo de controle, e o segundo com dois grupos experimentais e dois grupos placebo. As atividades dos grupos placebo foram formu- 
ladas para que os grupos não ficassem propriamente inativos, mas que suas atividades fossem tão leves que os seus membros não pudessem ser caracterizados como pessoas ativas. Os sujeitos dos grupos experimentais realizaram atividades físicas sistematizadas. No primeiro estudo participaram de um programa de exercícios um total de 24 adultos (17 mulheres e sete homens) entre 25 e 50 anos, durante seis meses. Os exercícios para o desenvolvimento de flexibilidade, coordenação, força e resistência duraram cerca de 60 minutos, uma vez por semana, com a intensidade podendo atingir cargas submáximas (75\% da carga máxima). Para a avaliação do programa, os pesquisadores utilizaram escalas criadas por eles mesmos, que incluíram (a) a autoavaliação positiva, (b) a autoavaliação negativa, (c) a aptidão física, e (d) a atratividade física. Usaram ainda a subescala SelfEsteem da Frankfurt Scales of Self-Concept (Deusinger, 1986) para avaliar a auto-estima, a versão alemã do Spielberger's State-Trait Anxiety Inventory (Laux, Glanzmann, Schaffner, \& Spielberger, 1981) para avaliar a ansiedade-traço e para avaliar as queixas psicossomáticas usaram a escala Beschwerde-Liste (Zerssen, 1976).

Os resultados deste experimento indicaram que as alterações mais significativas foram observadas principalmente nas variáveis associadas às experiências físicas, como autoconceito físico. As variáveis de ansiedade-traço e de autoestima permaneceram inalteradas. Segundo os autores, essas duas variáveis podem ser menos suscetíveis a mudanças decorrentes do exercício físico por serem características da personalidade do sujeito, mas um programa de exercício físico mais intenso (e.g., duas vezes por semana), pode produzir mudanças nas mesmas.

No experimento dois, 21 homens e 51 mulheres participaram de um programa de exercícios por seis meses. Neste experimento, Alfermann e Stoll (2000) dividiram os sujeitos da pesquisa em quatro grupos: dois experimentais e dois placebos. Um dos grupos experimentais praticou exclusivamente exercícios aeróbios - como caminhada, corrida, ciclismo e natação, duas vezes por semana, por 60 minutos, com intensidade adaptada a cada participante - não atingindo $70 \%$ da carga máxima individual. O outro grupo experimental seguiu a mesma proposta de exercícios que o grupo experimental do primeiro estudo (uma vez por semana durante uma hora, com exercícios de flexibilidade, coordenação, força e resistência). Um dos grupos placebo seguiu um programa de relaxamento muscular progressivo e treinamento autogénico. $\mathrm{O}$ segundo grupo placebo realizou alongamentos da cadeia posterior e aulas de correção postural, nas quais os sujeitos aprenderam a sentar-se e a movimentar-se de maneira a prevenir as dores nas costas. Ambos os grupos placebo tiveram encontros duas vezes por semana, com 60 minutos de duração. A duração total deste segundo estudo foi de seis meses. Basicamente, a forma de avaliação desta intervenção foi a mesma adotada no primeiro estudo. Foi feita apenas uma modificação no Spielberger's State-Trait Anxiety Inventory, ao qual se acrescentou uma subescala para avaliar traços de raiva. Testes de aptidões físicas também foram acrescentados para medir resistência, flexibilidade, equilíbrio, velocidade, força, arremesso à distância e coordenação. As avaliações atitudinais foram feitas em três momentos: no começo do programa de intervenção, ao final da intervenção e seis meses após a finalização do programa. Os testes físicos foram aplicados somente antes e logo após o final da intervenção.

Os resultados indicaram que os participantes dos grupos placebo tiveram melhores resultados do que os sujeitos dos grupos experimentais no que se refere a autoconceito e bem-estar e que os efeitos permaneceram estáveis seis meses após o término das atividades. Embora os participantes dos grupos experimentais de ambos experimentos terem tido ganhos significativos no que se refere a autoconceito físico depois de seis meses de exercício, apenas para o grupo experimental que praticou exercícios aeróbios (experimento 
dois) identificou-se incrementos de autoestima, e isso foi unânime entre os participantes. Para Alfermann e Stoll (2000), os resultados dos dois estudos confirmam a hipótese de que o exercício provoca melhorias no autoconceito físico; porém, não apenas os exercícios físicos, mas também outras atividades físicas, como as realizadas com os grupos placebo, são capazes de influenciar as variáveis dependentes.

Em outro estudo, Stoll e Alfermann (2002) analisaram os efeitos físicos na avaliação do autoconceito corporal e bem-estar entre idosos. Uma amostra de 88 pessoas, homens $(n=16)$ e mulheres $(n=72)$, com idade mínima igual a 50 anos foi recrutada para o estudo, que teve duração de 14 semanas. A amostra foi dividida em três grupos: (a) grupo experimental, que praticou exercícios moderados uma vez por semana durante 60-75 minutos; (b) grupo placebo, que teve aulas de línguas estrangeiras, uma vez por semana, durante 90 minutos; e (c) um grupo de controle, que não praticou atividades. A versão alemã do Conservation of Resources Evaluation (originalmente publicada por Hobfoll, 1989), quatro escalas criadas pelos autores para avaliar o autoconceito em idosos, a versão alemã do Spielberger's State-Trait Anxiety Inventory (Laux et al., 1981), e a escala Beschwerde-Liste (Zerssen, 1976) foram utilizadas antes do início e ao final da intervenção, para avaliar os efeitos do programa na amostra.

Os resultados revelaram melhorias no autoconceito corporal de homens e mulheres do grupo experimental quando comparado aos grupos de controle e placebo. As variáveis de recursos de avaliação e bem-estar subjetivo não apresentaram melhorias em nenhum dos grupos. Este estudo não identificou, entre os participantes dos três grupos, efeitos positivos para a ansiedade e para as queixas psicossomáticas. Stoll e Alfermann (2002) atribuíram esse achado às características dos exercícios, que não foram focados para tal objetivo, e consideraram também como fator a frequência semanal do programa - que seria menos do que o necessário para alterar as variáveis analisadas.
Ginis, Eng, Arbour, Hartman, e Phillips (2005) publicaram um estudo no qual examinaram as diferenças entre homens e mulheres, no que se refere à mudanças na imagem corporal e suas relações com as mudanças corporais subjetivas e objetivas. Participaram do estudo 25 homens e 16 mulheres sedentários que foram submetidos a um programa de treinamento resistido progressivo, com duração de 12 semanas e frequência de cinco dias por semana. Para avaliar o programa, os participantes responderam, antes e depois da intervenção, à (a) Social Physique Anxiety Scale (versão de 9 itens desenvolvida por Martin, Rejeski, Leary, McAuley, \& Bane, 1997), (b) à subescala Body Areas Satisfaction do Multidimensional Body-Self Relations Questionnaire (Brown, Cash, \& Mikulka, 1990), (c) às subescalas Body Fat e Body Strength do questionário Physical SelfDescription Questionnaire (Marsh, Richards, Johnson, Roche, \& Tremayne, 1994), (d) à escala Male Body Silhouette (Lynch \& Zellner, 1999) - para os homens - e (e) à escala Female Body Silhouette (Furnham, Titman, \& Sleeman, 1994) - para a mulheres.

Os resultados indicaram que homens e mulheres obtiveram respostas similares no que se trata de satisfação com áreas do corpo e demostraram um significativo aumento com a satisfação corporal e um decréscimo significante na ansiedade a partir da intervenção proposta. Apesar das semelhanças, as mudanças possuíram diferentes significados. Para os homens, as melhorias na imagem corporal se relacionaram apenas com as mudanças corporais subjetivas, como por exemplo a perceção da composição corporal, que nem sempre corresponde com a medida objetiva (em valores numéricos) desta variável. Para as mulheres, as mudanças na imagem corporal se relacionaram tanto com mudanças físicas subjetivas (perceção) como com mudanças objetivas (como aumento da força). Esses resultados sugerem que apesar de homens e mulheres obterem melhorias na imagem corporal provenientes do treinamento de força, eles se beneficiam do treino por diferentes razões. 


\section{DISCUSSÃO}

A leitura dos artigos selecionados nos permite inferir características, verificar uma evolução dos desenhos das pesquisas, desenvolver uma reflexão sobre as relações entre atividade física e imagem corporal e identificar os efeitos que mudanças no tipo de atividade, na intensidade, na duração e/ou na frequência podem exercer na imagem corporal.

Quanto às características da pesquisa em imagem corporal, nota-se, nos trabalhos aqui analisados, um grande foco na satisfação corporal. Dessa forma, fica evidente que a dimensão percetiva e as demais dimensões atitudinais da imagem corporal - afetos, crenças e comportamentos - estão subexplorados e uma compreensão ampliada da relação causal entre a atividade física e a imagem corporal, por ora, fica limitada.

Ainda em relação à pesquisa em imagem corporal, a análise dos artigos nos permitiu refletir sobre os instrumentos para avaliar a mesma. Thompson (2004) já havia afirmado que a variedade das escalas e as possibilidades de escolha são grandes atualmente, por isso é fundamental ao pesquisador delinear corretamente qual dimensão da imagem corporal ele pretende investigar e saber selecionar instrumentos com boas qualidades psicométricas. Entretanto, o estudo de Varray (1988) nos aponta para um outro fator: é importante considerar se o instrumento é adequado, não só em termos psicométricos, mas também se é sensível o suficiente para identificar as mudanças provocadas pelo exercício na imagem corporal. Esta análise da validade preditiva de uma escala é rara em nossa área, sendo de nosso conhecimento apenas um estudo que abordou este tipo de validade (Litt \& Dodge, 2008). Um projeto piloto pode ser uma solução para o pesquisador para testar seus instrumentos.

Quanto ao desenho da pesquisa, em uma análise cronológica, podemos notar um desenvolvimento importante no que se refere à metodologia e aos recursos de avaliação da imagem corporal. Nos estudos de Vanderey- cken et al. (1987) e Varray (1988), a ausência de um grupo de controle deixa o estudo vulnerável e sem parâmetros para analisar as mudanças ocorridas antes e depois da intervenção realizada com os sujeitos. A partir do estudo de Tucker e Mortell (1992), o grupo de controle se faz presente em todas as outras pesquisas. Além do grupo de controle, Stoll e Alfermann $(2000,2002)$ introduzem em suas pesquisas um grupo placebo, cujos dados permitiram novas conclusões.

Outra evolução no delineamento destas pesquisas sobre atividade física e imagem corporal foi a introdução de diferentes exercícios nas intervenções realizadas. Da terapia corporal (Vandereycken et al., 1987) ao treinamento com pesos (Tucker \& Maxwell, 1992) surgiram novas possibilidades tanto no planejamento quanto na execução da pesquisa em si, pois o espaço exigido por uma intervenção com pesos é menor. Especialmente para as intervenções longitudinais, os exercícios com pesos em ambiente fechado podem ser uma escolha coerente para países com inverno rígido, a fim de minimizar os riscos de dropout da amostra. Além de inovar os estudos, Tucker e Maxwell (1992) ainda apontaram para a variabilidade de efeitos que os diferentes tipos de exercício podem ter na imagem corporal, ampliando também a possibilidade de compreender mais amplamente a relação entre imagem corporal e cada tipo de exercício.

A escolha das atividades a serem realizadas na intervenção é, assim, de extrema importância. Adicionalmente, o estudo de Stoll e Alfermann (2000) aponta para uma reflexão interessante: os autores verificaram que $o$ grupo experimental - que realizou atividades de fitness duas vezes por semana - obteve melhores resultados para autoconceito físico quando comparados ao grupo placebo. Entretanto, os grupos placebos - que realizaram atividades de relaxamento e posturais também apresentaram melhorias significativas para as variáveis autoestima e bem-estar. Esses resultados nos levam a refletir sobre o contato com o corpo, provocado pelo movimento, que 
pode ser a matriz do desenvolvimento de uma nova relação e representação do corpo. Por tanto, os objetivos a serem alcançados através de um programa de treinamento, exigem um planejamento delicado da intervenção, já que os diferentes movimentos e intensidades provocam reações específicas, singulares a cada sujeito. Encontramos eco nessas reflexões no estudo de Calogero e Pedrotty (2004). Para os autores, planejar atenciosamente as atividades e determinar os objetivos do programa de exercícios é de extrema importância para gerar resultados positivos na imagem corporal da população alvo. Os autores planejaram atividades físicas para reduzir o abuso de exercícios físicos por mulheres com transtornos alimentares. Aqui, mais do que a frequência e a intensidade, o modo através do qual as atividades foram conduzidas fez a diferença para o estudo, que teve resultados positivos.

Além do tipo de exercício, o pesquisador deve considerar outros três fatores: frequência, intensidade e duração. Nas pesquisas aqui analisadas, pode-se observar que a frequência das intervenções variou de uma a cinco vezes semanais e a duração da sessão de 45 a 75 minutos. A intensidade dos exercícios empregados nas intervenções variou de leve a moderada - por volta dos $70 \%$ da frequência cardíaca máxima ou $75 \%$ da carga submáxima de trabalho. Todos os estudos, independente da duração da sessão (45 ou 75 minutos) identificaram melhorias significativas na imagem corporal de suas respetivas populações, com exceção dos estudos que realizaram suas práticas apenas uma vez por semana. Nesses estudos, a ansiedade, as queixas psicossomáticas, a autoestima e o bem-estar não sofreram alterações (Stoll \& Alfermann 2000, 2002).

Podemos também chamar atenção ao significado das atividades para os diferentes sexos. Ginis et al. (2005) enfatizam que homens e mulheres podem se beneficiar de atividades físicas, mas o fazem por razões diferentes. As mulheres dão maior importância aos resultados objetivos (referentes à medidas corpo- rais) e os homens atribuem um maior significado à perceção dos resultados obtidos, dando mais importância ao caráter subjetivo do mesmo. Ainda sobre esta questão, ressalta-se que a maioria dos estudos aqui analisados foram intervenções em amostras femininas. A área, que sofre com uma carência de estudos, fica ainda mais desfalcada de dados sobre outras populações e juntamente com a questão do foco quase exclusivo na avaliação da satisfação, ressaltada no segundo parágrafo, se constitui uma lacuna importante na literatura. Outra lacuna se refere aos adolescentes, pois nenhum estudo sobre este grupo amostral foi selecionado, pelos critérios de inclusão e exclusão, para esta revisão. Ademais, estudos com crianças também são escassos.

Finalmente, outra questão interessante está no planejamento da coleta de dados, que pode possibilitar - ou não - conclusões sobre alterações de traço ou estado - e sobre os limites do papel da atividade física na imagem corporal. Stoll (2000) e Alfemann e Stoll (2000) afirmam que os efeitos das atividades realizadas são significantes logo após a intervenção, apontada para uma imagem corporal mais positiva, mas desaparecem com o tempo, se os estímulos cessam. Estes resultados apontam se as alterações foram momentâneas (estado) ou permanentes (traço). Ressalta-se que toda experiência corporal é relevante numa aula de educação física, entretanto, pesquisas com diferentes amostras e planejamentos de intervenção são necessárias, para esclarecer se as alterações na imagem corporal, provocadas pela atividade física, são temporárias por natureza ou se essa característica varia de acordo com o tipo de intervenção.

A estas observações, gostaríamos de acrescentar o que ressalta Tavares (2003) em relação ao profissional de educação física que pretende trabalhar com o propósito de facilitar o desenvolvimento da imagem corporal do outro: este deve ter a sua própria imagem corporal bem desenvolvida, já tendo lidado com suas perdas, reconhecendo suas potencialidades e limitações. Dessa forma conseguirá se 
colocar na linha tênue entre a distância que abandona e o perto que sufoca, dando espaço para o outro identificar-se como sujeito, a partir do "ouvir" empático. Este profissional também deve considerar em seu planejamento a escolha das atividades, o objetivo que se pretende alcançar, a individualidade do sujeito (que não é apenas biológica, mas emocional) a duração da intervenção e frequência das sessões.

Para que a pesquisa em imagem corporal torne-se ainda mais completa, Campbell e Hausenblas (2009) sugerem que o próximo passo é investigar como os aspetos biológicos, fisiológicos e sociais do exercício contribuem para desenvolver a imagem corporal. Assim, as intervenções poderão ser projetadas de maneira mais eficaz e cuidadosa para que o exercício não venha a ser uma intervenção prática e acessível para a imagem negativa do corpo.

\section{Agradecimentos:}

Os autores agradecem ao CNPq, pela bolsa de fomento concedida.

\section{Conflito de Interesses:}

Nada a declarar.

\section{Financiamento:}

Os autores declaram que a agência de fomento CNPQ financiou esta pesquisa através de uma bolsa de estudos de iniciação científica (COG) doutorado à autora ANNBC.

\section{REFERÊNCIAS}

*Alfermann, D., \& Stoll, O. (2000). Effects of physical exercise on self-concept and well-being. Journal of Sport Psychology, 31 (1), 47-65.

Assunção, S. S., Cordás, T. A., \& Araújo, L. (2002). Atividade física e transtornos alimentares. Revista de Psiquiatria Clínica, 29, 4-13.

Brown, T. A., Cash, T. F., \& Mikulka, P. J. (1990). Attitudinal body image assessment: Factor analysis of the Body-Self Relations Questionnaire. Journal of Personality Assessment, 55, 135144. doi: 10.1207/s15327752jpa5501\&2_13

Bruchon-Schweitzer, M. (1987). Dimensionality of the body image: The Body Image Questionaire.
Perceptual and Motor Skills, 65, 887-892. doi: 10. 2466/pms.1987.65.3.887

Campbell, A., \& Hausenblas, H. A. (2009). Effects of exercise interventions on body image: A meta-analysis. Journal of Health Psychology, 14(6), 780-793. doi: 10.1177/1359105309338977

*Calogero, R. M., \& Pedrotty, K. N. (2004). The practice and process of healthy exercise: An investigation of the treatment of exercise. Eating Disorders, 12, 273-291. doi: 10.1080/106 40260490521352

Caspersen, C. J., Powell, K. E., \& Christensen, G. M. (1985). Physical activity, exercise, and physical fitness: Definitions and distinctions for health related research. Public Health Reports, 100(2), 126-131.

Cash, T. (2004). Body image: Past, present, and future. Body Image, 1, 1-5. doi: 10.1016/S17401445(03)00011-1

Cash, T., \& Pruzinsky, T. (2002). Body image: A handbook of theory, research, and clinical practice. New York: Guilford Press.

Clinton, D. (2001). Expectations and experiences of treatment in eating disorders. Eating Disorders, 9, 361-371.

Coopersmith, S. (1984). Manuel de l'inventaire d'estime de soi. Paris: Editions du Centre de Psychologie Appliquée.

Deusinger, I. (1986). Die Frankfurter Selbstkonzeptskalen (FSKN). Göttingen: Hogrefe.

Dupuy, H. J. (1970). Developmental rationale, substantive, derivative and conceptual relevance of the general well-being schedule. Fairfax. VA: National Center for Health Statistics.

Fairburn, C. G., \& Wilson, G. T. (1993). Binge eating: Nature, assessment, and treatment. New York: Guilford Press.

Fisher, S., \& Cleveland, S. (1958). Body image and personality. New York: Dover Press.

Fox, K. R., \& Wilson, P. M. (2008). Self perceptual systems and physical activity. In T. S. Horn, (Ed.), Advances in sport psychology (pp. 49-64). Champaign, Il: Human Kinetics.

Franzoi, S. L., \& Shields, S. A. (1984). The Body Esteem Scale: Multidimensional structure and sex differences in a college population. Journal of Personality Assessment, 48, 173-178. doi: 10.12 07/s15327752jpa4802_12

Furnham, A., Titman, P., \& Sleeman, E. (1994). Perception of female body shapes as a function of exercise. Journal of Social Behavior and Personality, 9, 335-352. 
Garner, D. M., Olmste, M. P., \& Polivy, J. (1983). Development and validation of a multidimensional eating disorder inventory for anorexia nervosa and bulimia. International Journal of Eating Disorders, 2(2), 15-33. doi: 10.1002/10 98-108X(198321)2:2<15::AID-EAT226002020 3>3.0.CO;2-6

${ }^{*}$ Ginis, K. A., Eng, J. J., Arbour, K. P., Hartman, J. W., \& Phillips, S. (2005). Mind over muscle? Sex differences in the relationship between body image change and subjective and objective physical changes following a 12-week strengthtraining program. Body Image, 2, 363-372. doi: 10.1016/j.bodyim.2005.08.003

Hausenblas, H., \& Fallon, E. A. (2006). Exercise and body image: A meta-analysis. Psychology and Health, 21 (1), 33-47. doi: 10.1080/1476832050 0105270

Hobfoll, S. E. (1989). Conservation of resources: A new attempt at conceptualizing stress. American Psychologist, 44, 513-524. doi: 10.1037/0003-06 6X.44.3.513

Laux, L., Glanzmann, P., Schaffner, P., \& Spielberger, C. D. (1981). STAI Das State-Trait Angst Inventar: Theoretische grundlagen und handweisung. Weinheim: Beltz Testgesellschaft.

Litt, D., \& Dodge, T. (2008). A longitudinal investigation of the drive for muscularity scale: Predicting use of performance enhancing substances and weightlifting among males. Body Image, 5, 346-351. doi: 10.1016/j.bodyim.200 8.04.002

Lynch, S. M., \& Zellner, D. A. (1999). Figure preferences in two generations of men: The use of figure drawings illustrating differences in muscle mass. Sex Roles, 40, 833-843. doi: 10.10 23/A:1018868904239

Marsh, H. W., Richards, G. E., Johnson, S., Roche, L., \& Tremayne, P. (1994). Physical selfdescription questionnaire: Psychometric properties and a multitrait - multimethod analysis of relations to existing instruments. Journal of Sport \& Exercise Psychology, 16, 270-305.

Martin, K. A., Rejeski, W. J., Leary, M. R., McAuley, E., \& Bane, S. M. (1997). Is the Social Physique Anxiety Scale really multidimensional? Conceptual and statistical arguments for a unidimensional model. Journal of Sport \& Exercise Psychology, 19, 360-368.

McKinley, N., \& Hyde, J. (1996). The objectified body consciousness scale: Development and validation. Psychology of Women Quarterly, 20,
181-215. doi: 10.1111/j.1471-6402.1996.tb004 67.x

McNair, D. M., Lorr, M., \& Droppelman, L. F. (1971). Profile of Mood States: Manual. San Diego, CA: Educational and Testing Service.

*Pinto, B. M., Clark, M. M., Maruyama, N. C., \& Feder, S. I. (2003). Psychological and fitness changes associated with exercise participation among women with breast cancer. PsychoOncology, 12, 118-126. doi: 10.1002/pon.618

Rosen, J. C., Orosan, P., \& Reiter, J. (1995). Cognitive behavior therapy from negative body image in obese women. Behavior Therapy, 26, 25-42. doi: 10.1016/S0005-7894(05)80081-4

*Stoll, O. (2000). Gesundheitssport und ressourceneinschätzungen. Sportwissenschaftliche Beiträge, $41(1), 127-147$.

*Stoll, O., \& Alfermann, D. (2002). Effects of physical exercise on resources evaluation, body self-concept and well-being among older adults. Anxiety, Stress, and Coping, 15(3), 311319.

Tavares, M. C. (2003). Imagem corporal: Conceito e desenvolvimento. Barueri, SP: Manole.

Tavares, M. C. (2007) (Org). O dinamismo da imagem corporal. São Paulo: Phorte.

Thompson, J. K. (1990). Body image disturbance: Assessment and treatment. New York: Pergamon Press.

Thompson, J. K. (2004). The (mis) measurement of body image: Ten strategies to improve assessment for applied ad research purposes. Body Image, 1, 7-14. doi: 10.1016/S1740-1445(03)00 004-4

Thompson, J. K., \& Gardner, R. (2002). Measuring perceptual body image among adolescents and adults. In T. Cash, \& T. Pruzinsky (Eds.), Body Image: A handbook of theory, research \& clinical practice (pp. 135-141). New York: Guilford.

Thompson, J. K., \& Pasman, L. (1991). The Obligatory Exercise Questionnaire. Behavior Therapist, 14, 137.

Tucker, L. (1981). Internal structure, factor satisfaction, and reliability of the Body Cathexis Scale. Perceptual and Motor Skills, 53, 891-896.

*Tucker L. A., \& Maxwell, K. (1992). Effects of weight training on the emotional well-being and body image of females: Predictors of greatest benefit. American Journal of Health Promotion, 6(5), 338-371.

*Tucker, L. A., \& Mortell, R. (1993). Comparison of the effects of walking and weight training 
programs on body image in middle-aged women: An experimental study. American Journal of Health Promotion, 8(1), 34-42.

*Vandereycken, W., Depreitere, L., \& Probst, M. (1987). Body-oriented therapy for anorexia nervosa patients. American Journal of Psychoterapy, 41 (2), 252-259.

Vandereycken, W., \& Meermann, R. (1984) Anorexia nervosa: A clinician's guide to treatment. New York: Walter de Gruyter.

*Varray, A. (1988). Role d'une pratique physique adaptée sur la problématique de la représentation de soi et de l'image du corps. Revue des
Sciences et Techniques des Activités Physiques et Sportives, 9(8), 33-45.

Watson, D., Clark, L. A., \& Tellegen, A. (1988). Development and validation of brief measures of positive and negative affect: The PANAS scales. Journal of Personality and Social Psychology, 54, 1063-1070. doi: 10.1037/0022-3514.54.6.1 063

Zerssen, D. V. (1976). Die Beschwerdenliste. Weinheim: Beltz Testgesellschaft.

* As referências marcadas com asteriscos indicam os artigos que fizeram parte do resultado da revisão bibliográfica.

(c) EY-NC Todo o conteúdo da revista Motricidade está licenciado sob a Creative Commons, exceto quando especificado em contrário e nos conteúdos retirados de outras fontes bibliográficas. 